\title{
An Interactive RADIANCE Toolkit for Customizable CT Dose Monitoring and Reporting
}

\author{
Tessa S. Cook • Anand Sundaram • William W. Boonn • \\ Woojin Kim
}

Published online: 29 January 2013

(C) Society for Imaging Informatics in Medicine 2013

\begin{abstract}
The need for tools to monitor imaging-related radiation has grown dramatically in recent years. RADIANCE, a freely available open-source dose-monitoring tool, was developed in response to the need for an informatics solution in this realm. A number of open-source as well as commercial solutions have since been developed to enable radiology practices to monitor radiation dose parameters for modalities ranging from computed tomography to radiography to fluoroscopy. However, it is not sufficient to simply collect this data; it is equally important to be able to review it in the appropriate context. Most of the currently available dose-monitoring solutions have some type of reporting capability, such as a real-time dashboard or a static report. Previous versions of RADIANCE have included a real-time dashboard with pre-set screens that plot effective dose estimates according to different criteria, as well as monthly scorecards to summarize dose estimates for individuals within a radiology practice. In this work, we present the RADIANCE toolkit, a customizable reporting solution that allows users to generate reports of interest to them, summarizing a variety of metrics that can be grouped according to useful parameters. The output of the toolkit can be used for real-time dose monitoring or scheduled reporting, such as to a quality assurance committee. Making dose parameter data more accessible and more meaningful to the user promotes dose reduction efforts such as regular protocol review and optimization, and ultimately improves patient care by decreasing unnecessary radiation exposure.
\end{abstract}

T. S. Cook $(\bowtie) \cdot$ W. W. Boonn $\cdot$ W. Kim

Department of Radiology, Hospital of the University

of Pennsylvania, 1 Silverstein 3400 Spruce Street,

Philadelphia, PA 19104, USA

e-mail: tessa@alumni.upenn.edu

A. Sundaram

Department of Bioengineering, University of Pennsylvania School

of Engineering and Applied Sciences, Philadelphia, PA, USA
Keywords Radiation dose monitoring · Dose reduction . RADIANCE

\section{Background}

CT utilization has increased significantly in the last decade [1, 2]. In the last two decades, the proportion of background radiation in the USA attributed to medical imaging has increased from approximately $15 \%$ in 1987 to nearly $50 \%$ today $[3,4]$. Exposure to radiation as a result of medical imaging continues to be in the spotlight, receiving attention from professional organizations such as the American College of Radiology (ACR), Society of Pediatric Radiology (SPR) and the American Association of Physicists in Medicine (AAPM), and more notably, from the US House of Representatives Subcommittee on Health [5], as well as the lay press [6, 7]. Since July 2012, radiologists in the state of California have been required to include dose parameter information in their CT reports [8].

Both the ACR and SPR are strongly promoting dose monitoring via their ImageWisely ${ }^{\mathrm{TM}}$ and ImageGently ${ }^{\mathcal{O}}$ campaigns, respectively. In addition, the ACR's Dose Index Registry is actively collecting data from imaging centers worldwide, in order to ultimately develop dose reference levels and benchmarks for specific CT examinations [9]. Furthermore, there are regional efforts underway in various parts of the USA to promote local as well as internal dose monitoring [10]. A number of commercial and open-source solutions for CT dose monitoring and reporting have been developed in recent years. Among them is RADIANCE, a free, open-source package that uses optical character recognition to extract dose-related parameters from image-based dose sheets, but also imports dose parameters from the new radiation dose structured report [11].

One of the features of RADIANCE is a dashboard with predefined views of dose parameters according to departmental section, scanner type, involved personnel or individual patients. While users have found that the dashboard augments 
their ability to monitor CT dose parameters, they have often expressed a desire to be able to customize the individual dashboard screens or generate new reports. This is possible in the current implementation if the user is familiar with databases and query design, and willing to navigate the built-in data viewer provided with the database management system used by RADIANCE. However, users without database experience are limited to the provided dashboard screens.

Routine dose monitoring is critical in motivating protocol optimization and dose reduction. However, the large number of $\mathrm{CT}$ examinations performed at a given imaging facility can make manual dose reporting prohibitive. In addition, every imaging facility has unique needs when it comes to $\mathrm{CT}$ dose reporting. To that end, we present the RADIANCE toolkit, which enables imaging facilities to produce customized tables or reports according to their individual dose reporting and review needs.

\section{Methods}

The toolkit is built on the RADIANCE database schema and enables users to produce a wider variety of customized dose reports that take advantage of the data collected by RADIANCE. The toolkit is implemented using PHP, HighCharts (a full-featured JavaScript graphing library), DataTables (a JavaScript table library), as well as jQuery (a family of JavaScript libraries for visualization and interactive tasks). A simple user interface allows for interactive generation of either charts or tabular reports, without requiring prior knowledge of databases, query language or even the RADIANCE database schema.

Users can specify the $x$ - and $y$-axes of the desired chart by selecting data elements from the RADIANCE database from provided menus. Data points can be grouped according to criteria specified in drop-down boxes. Both quantitative (e.g., total study dose-length product) and categorical (e.g., scanner ID) variables are included as options for charting and grouping. The dependent variables that can be displayed include estimated whole-body effective dose, total study dose-length product (DLP), total study tube current-time product, and mean and maximum volume $\mathrm{CT}$ dose index $\left(\mathrm{CTDI}_{\mathrm{vol}}\right)$. Independent variables include time, patient demographics (age, height, weight), and scanner features (manufacturer, model, station name). Grouping criteria include scanner features, institution name, and patient gender. Using such parameters, the toolkit generates interactive charts in real time by applying the user's specifications to the data stored in the RADIANCE database. These charts can be printed or exported for inclusion in other reports.

Some display parameters are automatically defined based on the user's inputs. By default, the most recent month's worth of data is displayed, however, any start and end dates can be specified. The chart type is automatically assigned based on the combination of parameters chosen for the chart axes and the grouping criterion. For example, when numeric quantities such as time or patient demographic data are chosen as the independent variable, a scatter plot is used by default to show individual dose parameter values. Instead, when the independent variable is categorical, such as a scanner type or institution name, the average dose parameter is displayed using a column plot.

In addition to graph generation, users can also perform database queries with the same user interface and produce tables that can be exported as comma-separated values (CSV) or copied directly into a spreadsheet. The toolkit obviates the need to learn MySQL, the query language used in RADIANCE, or to understand the RADIANCE database schema, in order to craft and submit certain database queries. The exported data can then be imported into external spreadsheet or database software for further analysis or scheduled reporting. Alternatively, a printer-friendly tabular format can also be generated directly from the toolkit.

\section{Results}

Figure 1 shows the RADIANCE toolkit interface. The top panel of the toolkit displays the chart/report creation options: variable selection, time interval, and grouping parameter. In this case, we have chosen to review dose parameters for single-phase abdomen/pelvis CTs during the month

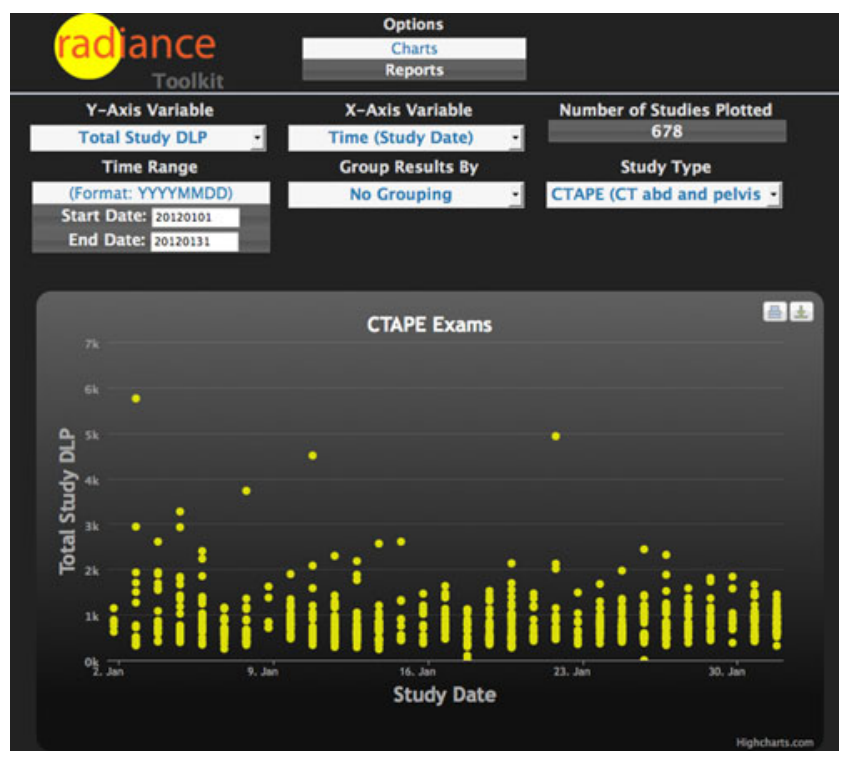

Fig. 1 Screen shot from the charting page of the RADIANCE toolkit, demonstrating 1 month's worth of dose parameters for single-phase abdomen/pelvis CTs. This chart is created with the default settings of total study DLP on the $y$-axis, time on the $x$-axis, no grouping parameter, and 1 month of data. The user must specify the study type; the chart type is automatically chosen. The chart can be printed or saved as an image using the icons at the top right of the chart pane 
of January. The chart in the bottom panel is automatically refreshed when new parameters are chosen and shows that most of the DLP values are clustered under $2,000 \mathrm{mGycm}$, with a few studies exceeding that level. In addition, the two icons in the top right corner of the chart can be used to print or save the chart, respectively, for inclusion in a larger quality improvement/dose monitoring report.

Figure 2 demonstrates the grouping option, showing the same data now classified by the institution at which the study was performed. Figure 3 demonstrates a review of average dose estimates for this same cohort of studies according to the scanner model on which the studies were performed. Both positive (lower than expected) and negative (higher than expected) outliers can be identified using this analysis for further investigation and review. The MySQL query required to produce the data for the chart in Fig. 3 is as follows: SELECT ManufacturersmodelName, AVG (CalculatedTotalDLP), COUNT (AccessionNumber) FROM masterflatfile WHERE CodeValue = 'CTAPE' AND StudyDate $>=20120101$ AND StudyDate $<=20120131$ AND EstimatedDose > O GROUP BY ManufacturersModelName ORDER BY ManufacturersModelName. While this is not an inherently complex query, it does require a working knowledge of query language to assemble. Instead, the RADIANCE toolkit can automatically assemble the query, organize the data and display it in a user-friendly environment.

In addition to its charting capabilities, the toolkit also provides tools for large-scale data query and exporting.

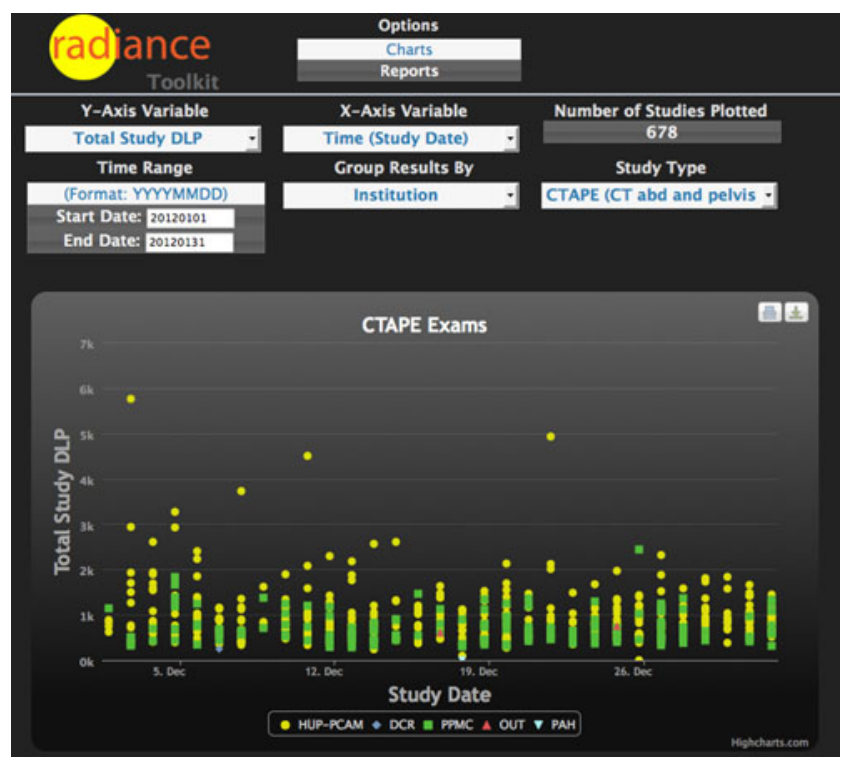

Fig. 2 An example of the grouping option applied to the data in Fig. 1. Here, the dose parameters are color coded according to the institution at which the study was performed. Other grouping options currently available include patient gender and scanner parameters such as manufacturer, model, and station name

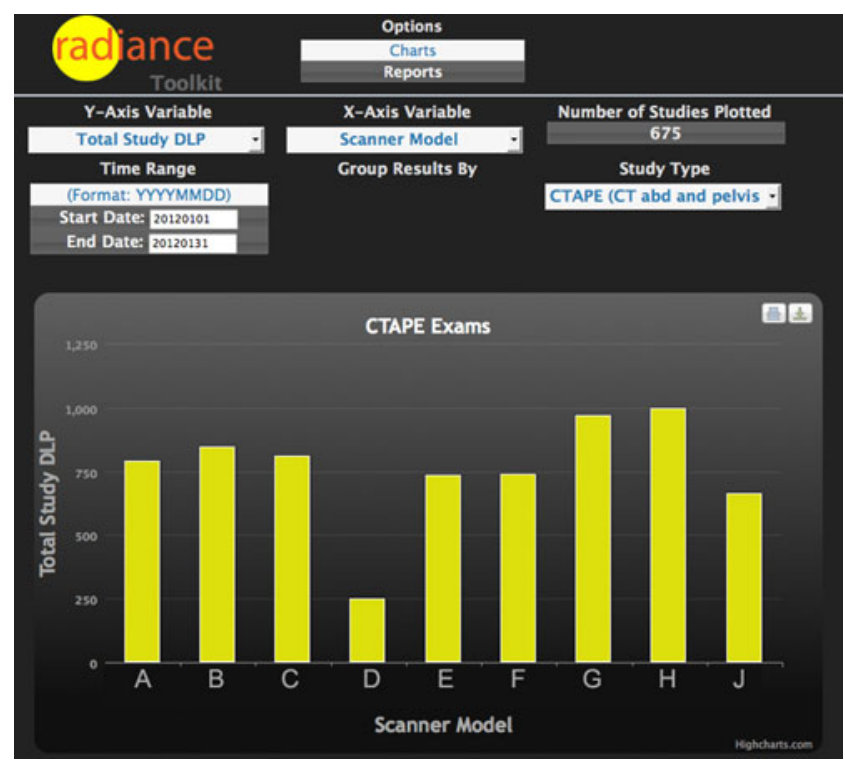

Fig. 3 Average dose parameters plotted by scanner model (anonymized). This chart was produced using the same data from Figs. 1 and 2, but now allows direct comparison of data from different scanners for the same type of study

Figure 4 shows the export screen with a filter for the study type at the top of the screen that retrieves all summary data for a particular study type during a time interval stored in the RADIANCE database. Buttons in the bottom left hand corner of the table (not shown) provide options for copying and pasting the data or exporting to CSV or Microsoft Excel files for further analysis offline. Figure 5 shows the same plot as in Fig. 3, generated from the exported data shown in Fig. 4 rather than within the toolkit itself. Using spreadsheet software, this plot required approximately two dozen mouse clicks and additional typed input to reproduce from the exported data, as opposed to less than half a dozen mouse clicks with the RADIANCE toolkit.

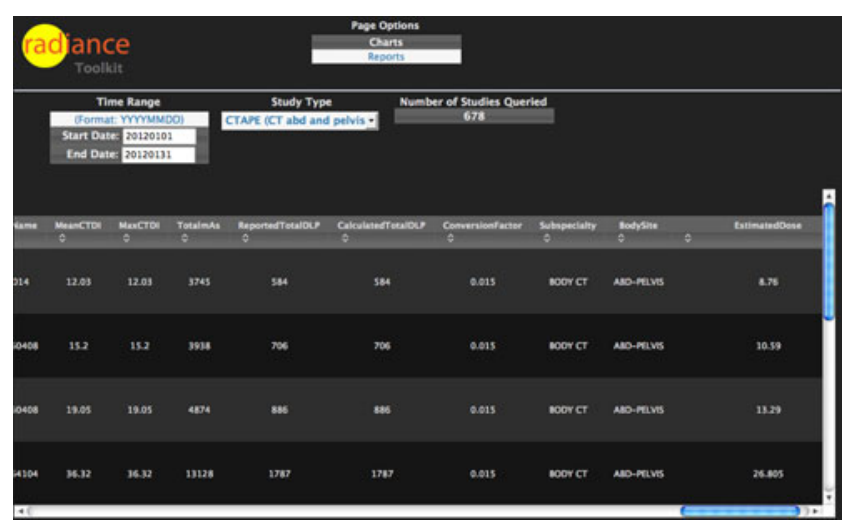

Fig. 4 Screen shot from the export page of the RADIANCE toolkit, demonstrating the filter options (date and study type) at the top of the page and the results of the automatically formulated query at the bottom. This data can be copied and pasted into a spreadsheet or exported as a CSV or Microsoft Excel file for further offline analysis 
Fig. 5 The same analysis as in Fig. 3, generated with spreadsheet software instead of the RADIANCE toolkit using the exported data shown in Fig. 4
CTAPE Exams

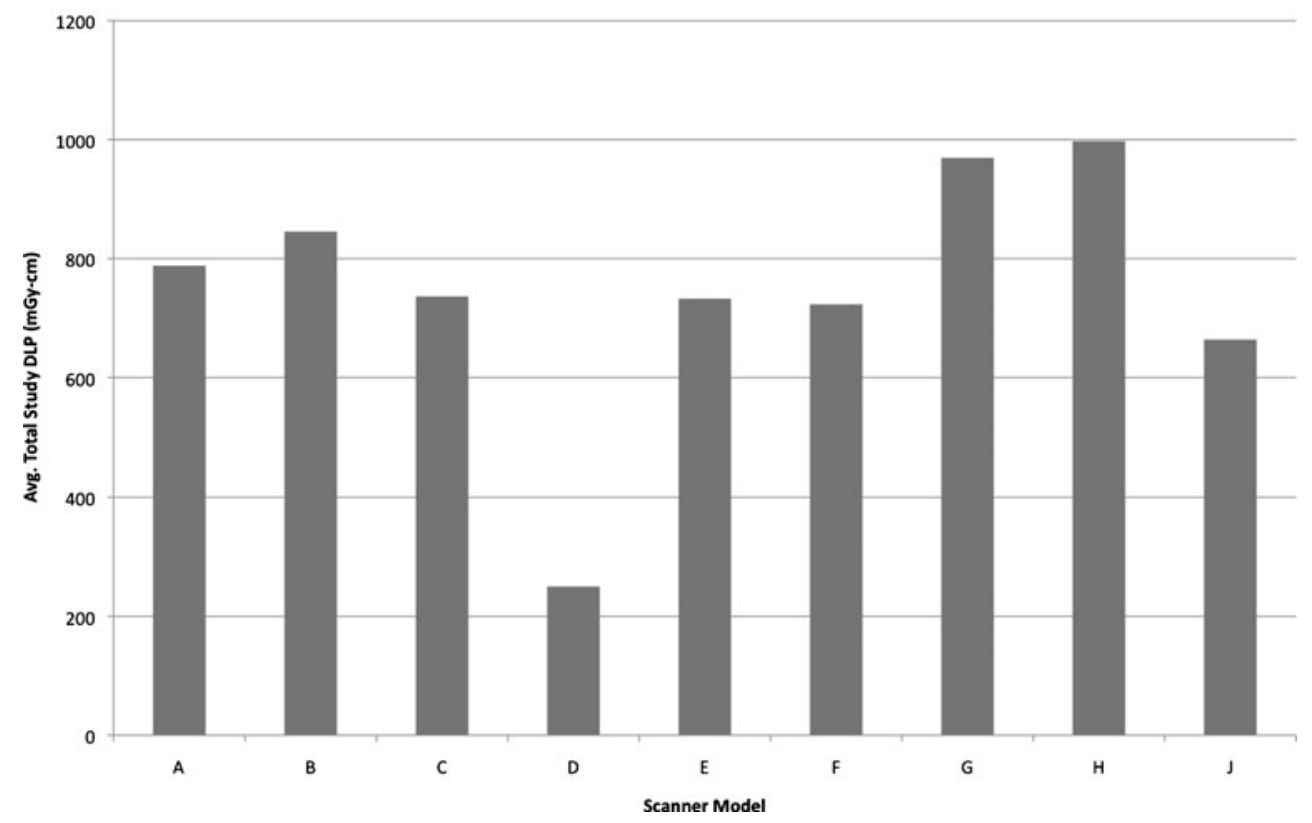

\section{Discussion}

With the increasing emphasis being placed on radiation dose monitoring and protocol optimization, it is important for imaging facilities to have policies in place for dose tracking, outlier identification and analysis of dose estimates. While all of these tools are available in the version of RADIANCE currently available, they are constrained by the predefined screens and reports implemented in the real-time dashboard and monthly scorecards. The RADIANCE toolkit is an extension of these reporting capabilities that puts more control into the users' hands. This is singularly important, as individual imaging facilities have unique dose monitoring and reporting needs, and the ability to customize report generation becomes important. With the toolkit, users are able to look at how dose varies with a number of specific factors, such as patient weight, patient age, or the particular scanner used. Charts and tables with this analysis can easily be generated for inclusion in larger reports, such as to a facility's quality improvement committee.

In this initial version of the toolkit, only one grouping parameter is allowed. This limitation was chosen to produce simple, straightforward graphs and decrease the number of decisions required of the user. In addition, the choice of how to plot the dependent variable (e.g., scatter vs. column plot) was made automatically based on whether the user selected a quantitative or categorical parameter as the independent variable. Future versions will likely include the ability for the user to customize the chart type as well as the associated choice of average versus actual dependent variable.
For the toolkit to become useful as a quality control/quality assurance tool, it is conceivable that a facility's quality and safety committee may wish to generate the same report each month to evaluate the previous month's dose estimates. This would mean creating the same query each month and generating a chart or report. While this process is easier now with the toolkit, one future feature of note would be the ability to save or "bookmark" queries for ease of reproduction later. Generation of month-to-month comparisons or evaluation of annual trends is already possible with the scorecards, another reporting tool already available within the RADIANCE distribution. Additional future work would include a suite of dose analytics tools, with the ability to generate multiple charts and/or tables as part of a customized report.

The toolkit is available for download with RADIANCE version 2.5 (http://www.radiancedose.com). In general, RADIANCE requires no sophisticated hardware and can be installed on any modern Windows-based personal computer. The authors recommend that the software be installed on a standalone computer or server that is not running PACS or another clinical application that requires intensive computing resources. All the software components required to run RADIANCE are included in the installation file.

Upon installation, the computer or server running the software can be set up as a DICOM listener using a provided script. Either image-based dose sheets or radiation dose structured reports can then be sent from PACS or individual scanners by configuring this new DICOM node as a CMOVE destination on the PACS or each scanner. In addition, another script within RADIANCE is able to query certain flavors of PACS and retrieve image-based dose sheets. RADIANCE users who also participate in the 
American College of Radiology's Dose Index Registry (DIR) can not only track dose parameters internally but also send data to the DIR by separately installing their TRIAD Site Server application [9].

\section{Conclusion}

Radiation dose monitoring has become increasingly necessary in recent years. However, every imaging center has unique dose monitoring and reporting needs. The RADIANCE toolkit enables imaging facilities to produce customized dose reports with an interactive user interface, without having to understand the underlying intricacies of structured query language or a database schema. Use of the RADIANCE toolkit promotes regular dose monitoring and reporting and allows imaging facilities to improve care of their radiology patients.

\section{References}

1. Maitino AJ, Levin DC, Parker L, Rao VM, Sunshine JH: Nationwide trends in rates of utilization of noninvasive diagnostic imaging among the Medicare population between 1993 and 1999. Radiology 227:113-117, 2003

2. Levin DC, Rao VM, Parker L, Frangos AJ, Sunshine JH: Recent trends in utilization rates of abdominal imaging: the relative roles of radiologists and nonradiologist physicians. JACR 5:744-747, 2008

3. Kase KR, et al: "Ionizing radiation exposure of the population of the United States," no. 160, 2009

4. Sinclair WK, Adelstein SJ, Carter MW, Harley JH, Moeller DW: "Ionizing radiation exposure of the population of the United States," no. 93, 1987

5. Congressional Subcommittee on Health: "Medical radiation: an overview of the issues", 2010

6. Bogdanich W, Ruiz RR. "FDA to increase oversight of medical radiation," New York Times

7. Landro L: "Radiation risks prompt push to curb CT scans," Wall Street Journal

8. "SB 1237—California State Senate." Available: http://info.sen.ca. gov/pub/09-10/bill/sen/sb_1201-1250/sb_1237_bill_20100929_ chaptered.html

9. American College of Radiology, "ACR Dose Index Registry." Available: https://nrdr.acr.org/Portal/DIR/Main/page.aspx

10. ECRI Institute, "CT Scans: Right Dose, Right Now." Available: https://www.ecri.org/blog/Lists/Posts/Post.aspx?ID=102. [Accessed: 09-Sep-2011]

11. Cook TS, Zimmerman SL, Steingall SR, Maidment ADA, Kim W, Boonn WW: RADIANCE-An Automated, Enterprise-wide Solution for Archiving and Reporting CT Radiation Dose Estimates. Radiographics 31:1833-1846, 2011 